\title{
Several questions in differentiation of human embryonic stem cells to endothelial cells
}

\author{
Xuan Sun ${ }^{1}$, Lamei Cheng ${ }^{1}$, Guangxiu Lu ${ }^{1}$ \\ ${ }^{1}$ National Center of Human Stem Cell Research and Engineering; Institute of Human Reproduction and Stem Cell Engineering, \\ Central South University, China
}

There are accumulating knowledge and technologies in induction and identification of human embryonic stem cells(hESCs) derived endothelial cells. However, the variation of the results in different laboratories is large, and the reproducibility of the experiments is not reliable. There are three probable reason: 1 . The differences of hESC lines; 2. The differences of culture condition; 3. The differences of sorting markers that have been used to purify endothelial cell population. In this study, 5 normal hESC lines from Asian race were established and employed to differentiate into endothelial cells. After 9 days spontaneous differentiation of embryoid bodies (EBs), the percentage of KDR (17-45\%), VE-cadherin(0.2-0.9\%) and CD34(1.7-2.8\%) positive cells showed significant differences compared with previously reported results; but did not show significant differences among 5 cell lines. We isolated KDR+ cells from 9-day-old EBs by immunomagnetic beads sorting, these cells were plated in EGM-2 medium supplemented with vascular endothelial growth factor (VEGF). After 7 days culture, these cells expressed KDR, VE-cadherin, PECAM1, UEA1 but not mature endothelial cell protein vWF; and were capable of taking up DiI-Ac-LDL and forming capillary-like tube structure on matrigel, thus these cells are primitive endothelial-like cells. KDR derived endothelial-like cells showed the similar abilities of proliferation and capillary-like structure formation when compared with endothelial progenitor cells derived from cord blood. The results suggest that the KDR+ cells-derived cells are a kind of primitive endothelial-like precursors.

Keywords: hESCs, endothelial cells, differentiation, KDR

Cell Research (2008) 18:s126. doi: 10.1038/cr.2008.216; published online 4 August 2008

Correspondence: Guangxiu Lu

E-mail: lugxdirector@yahoo.com.cn 